\title{
La naturaleza de la creatividad
}

The nature of creativity

Recibido: 15/08/2020

Aceptado: 15/12/2020
Oscar Armando González Díaz

Juan Tzoc ${ }^{2}$

Julieta Maribel López ${ }^{3}$

Servio Tulio Argueta Ramos ${ }^{4}$

Correo: oscargondiaz3696@gmail.com

Filiación de los autores: Universidad de San Carlos de Guatemala

https://doi.org/10.36314/revistavida.v2i1.8

\section{Resumen}

La vida no es producto de algún fenómeno casual. La vida responde a un sistema complejo que está relacionado de manera universal desde el surgimiento de la materia y sus diferentes campos energéticos. De esa cuenta, se puede decir todo esta interconectado, el funcionamiento de un elemento de la naturaleza depende directamente con los demás elementos. Y ese patrón sigue en todas las relaciones en donde se ve involucrada la persona, el desarrollo de las sociedades se conecta a las realidades económica, ambiental, política, social y cada una de estas relaciones guarda una conexión de tipo fractal.

Esta situación permite comprender que el conocimiento se construye de manera compleja y no de manera lineal como tradicionalmente se piensa. Por lo tanto, cada pueblo, cada cultura y cada sociedad tienen su forma propia de ver y percibir la realidad. Cada generación de seres humanos tiene su propia cosmovisión que, al hacer reflexiones profundas, se nota cómo, cada una de las epistemologías, tiene sus semejanzas y coincidencias típicas, tales como las facultades que tienen todos los humanos en el funcionamiento del cerebro y el sistema nervioso.

Por lo tanto, cada ser humano y cada cultura es poseedor de grandes conocimientos, que son las expresiones creativas de los patrones que dieron origen a la naturaleza. Sistemas que se van repitiendo para dar vida a nuevas creaciones, que pueden servir para el crecimiento o destrucción de las formas de vida del universo, porque todos, forman parte del todo, y todo está relacionado en un sistema complejo que surge del caos.

\section{Palabras clave}

Bioética, cosmovisión, caos, complejidad, creatividad

1 MSc. en Docencia Universitaria con énfasis en Estrategias de Aprendizaje, Asesor Pedagógico del Sistema Nacional de Acompañamiento Educativo (SINAE)

2 MSc. en Educación Intercultural, docente de Investigación en Postgrado USAC CUNOR y consultor en MINEDUC.

3 M.A. en Medición, Evaluación e Investigación Educativas, consultora independiente.

4 MSc en Docencia Universitaria con énfasis en Andragogía, MSc. Administración de Recursos Humanos, Postgrado en Educación en Diabetes, Médico y Cirujano, Docente en USAC CUNORI en carrera de medicina. 


\begin{abstract}
Abstrac
The life itself is not a coincidence phenomenon. Life as a concept, is a complex process interelated with the energy flow chanels. From this point of view, every element action interacts with the whole element. Human beign has this similar pathway, the society development is directly related to environmental, economical, political and social relationsips conections aiming to a fractal connetion type. Understanding this situation, knowledge is coming from a complexity way and not from a linear one model, as some people said. Even though, every culture, town, society has its own way to appreciate the reality. Every human being generation becomes its own cosmovisión, when we do a deep analysis, we realice about simmilarities, typical coincidences aiming to develop brain and nervous system functionality.

So important to recognize, every human being, town, village, and cultural lifestyle is holder of amazing knowledge that the end, becomes the creative pathways of the nature origin. Pathways that become repetitive from generations, giving action to new creative ones, which may to enhance or destroy the people themselves, because everything is part of the one whole, and its whole is related a complex system coming from the caos.
\end{abstract}

\title{
Keywords
}

Bioethics, cosmovisión, caos, complexity, creativity

\section{Las construcciones sobre la verdad}

Actualmente el mundo enfrenta una serie de problemas y fenómenos que cada vez lo ponen al filo de los riesgo y contagios, que la misma humanidad ha participado en acelerar su propia destrucción, con el afán de manipular, alterar, minar y complicar la naturaleza de las cosas, es importante considerar la contribución del conocimiento en el presente donde vivimos.

La revolución científico-tecnológica contemporánea se manifiesta profundamente en la transformación de las acciones humanas en los ámbitos del saber y de la ciencia, y se expresa en la ruptura de los mismos saberes y de la ciencia del enfoque clásico. ${ }^{5}$

Los cambios propuestos por la ciencia clásica se han visto afectados por la emancipación de la complejidad y de la globalización que se está dando actualmente. Esto se refiere a que en las ciencias clásicas existen dos tipos de carencias cognitivas, siendo una de estas la ceguera del modo del conocimiento, cuando se segmentan los saberes y se propicia la desintegración de los problemas fundamentales, porque estos es su abordaje demandan el conocimiento interdisciplinar. Por otra parte, la segunda carencia cognitiva consiste en el occidental centralismo ubicado en el pedestal de la racionalidad, en una posición ilusoria de poder universal. ${ }^{6}$

5 Delgado, Carlos, "Hacia un nuevo saber. La bioética en la revolución contemporánea del saber", Universidad El Bosque. Kimpres, 2008. Pág. 7.

6 Morin, Edgar, "La Vía, para el futuro de la humanidad", Madrid: Paidós, 2011. Pág.19 
Por lo tanto, a criterio Morin, no solo es la ignorancia la que conduce a las personas a actuar y pensar de esta manera, sino también el reduccionismo en el conocimiento, que muchas veces no permite ver más allá del alcance de los ojos, además de parcializar lo que por naturaleza conlleva otras relaciones establecidas por las leyes naturales.

Por lo tanto, en la epistemología dialéctica, inspirado en las teorías de Marx, el sujeto cognoscente desarrolla la comprensión y aprehensión del mundo por la interacción con la totalidad de los objetos y de las estructuras delimitadas en la construcción de su mundo, que resultan de sus propias actividades ${ }^{7}$ dando valor predominante al saber científico y la tecnología, como justificación y fundamentación de la verdad, prevaleciendo de esta forma el enfoque reduccionista desde una única perspectiva y desde la interpretación absoluta de una única mirada, además de la comprensión segmentada desvinculada de las relaciones complejas de los fenómenos y elementos del universo. ${ }^{8}$

Se puede decir, que los valores provienen de la invención humana, asimismo, la idea de conocimiento objetivo. Observación, también compartida por otros filósofos como Focault, Maturana, Wttgenstein, entre otros; 9 en otras palabras, nos quiere decir que las ideas humanas son relativas, por tanto, no pueden considerarse absolutas y únicas porque parten de la experiencia personal y de la diversidad de experiencias que genera diversas interpretaciones, concepciones y construcciones tanto de los valores como de los conocimientos.

Es importante reconocer que Marx a diferencia de otros ponentes del pensamiento científico, en cuanto al materialismo se refiere, dista en sus ideas y concepto de materia en su sentido relacional, diferenciándose en el marco de la teoría dialéctica positivista.

Posteriormente, surgió el planteamiento de la revolución científica de Kuhn, basado en el paradigma más estructuralista, donde el conocimiento se considera fundamentado en estructuras concebidas por la comunidad científica, en las que el rol del sujeto es un segmento de un colectivo que produce el saber desde una perspectiva valorativa. ${ }^{10}$

Según este autor, en esta corriente de pensamiento, el modo de producir conocimiento fue desarrollado mediante el manejo de conceptos, hipótesis, teorías, leyes, guías o libros, en un método lineal y reduccionista que afectó la producción de conocimientos endógenos.

7 Delgado, Op cit., 2008. Pág. 62

8 Delgado, Op cit., 2008. Pág. 62.

9 lbídem., Pág. 63

10 Ibídem., Pág. 64

https://revistavidacunori.com 
Luego de esto, surge un nuevo debate epistemológico representado en la teoría de la complejidad, que ha propiciado la ruptura dicotómica entre el sujeto y el objeto promovida por el enfoque racional, modificando significativamente la concepción impuesta por la racionalidad clásica.

En esta corriente epistemológica Delgado identifica las propuestas que versan sobre la teoría de catástrofes, la cibernética del segundo orden, la teoría del caos, el geométrico fractal, las estructuras disipativas, el pensamiento complejo, entre otros; mismas que resultan revolucionarias por el aporte que brindan al avance de la ciencia y la evolución del pensamiento filosófico, metodológico y epistemológico que en la actualidad la producción el conocimiento requiere. ${ }^{11}$

El enfoque relacional, se distingue porque considera el mundo y los objetos desde una concepción del sujeto en correspondencia al entorno.

Asimismo, como surge la creatividad y el orden dentro del caos, igualmente otras relaciones como la que existe entre la inspiración y la planificación o bien entre el destino y el azar, donde la diversidad y la resonancia como los fractales son la manifestación en la naturaleza.

De esta manera compleja se construyen el pensamiento de los pueblos a partir de lo cotidiano, en la vivencia inmersa en la dimensión del espacio y del tiempo fractal, y en las turbulencias del caos emergente desarrollador de la cotidianeidad creativa.

El innovador enfoque desde la complejidad, es un abordaje epistemológico de segundo orden, y resalta un paradigma basado en tres líneas de trabajo, según Maldonado estas líneas de trabajo consisten: a) en concebir la complejidad como ciencia que aborda la dinámica no lineal de los sistemas concretos; b) en la complejidad como método de pensamiento, que supera la separación de las disciplinas del saber y se enfoca en el aprendizaje del pensamiento relacional; y c) en la complejidad y la cosmovisión, donde se construye un nuevo enfoque de ver al conocimiento y al mundo emergiendo de la reflexión holística del pensamiento sistémico. ${ }^{12}$

Por esa razón, cualquier enfermedad, cualquier mal o sufrimiento, son fenómenos que han servido para aprender y volver la mirada hacia atrás y retomar el camino que muchas veces se desvía, se abandona o se olvida; y cada vez que se olvida, se descuida o se altera, se rompe el equilibrio, se degrada y se vulnera a los demás seres de la naturaleza; algunos que dependen directamente de los otros,

11 Delgado, Op cit., 2008. Pág. 65

12 Ibídem., Pág. 82. 
mientras que otros, dependen relativamente en una cadena, como dice la teoría de los fractales. ${ }^{13}$

Morin, en relación al pensamiento complejo argumenta que, este rechaza la postulación del determinismo universal. Adiciona, que el universo no está sujeto de forma absoluta a un orden, el universo se encuentra inmerso en el juego con características de dialógica, antagonismo y complementariedad; en situaciones emergentes de orden, desorden y autoorganización. ${ }^{14}$

Este enfoque es el que ha impulsado Morin, caracterizado por la sistematicidad de la teoría del pensamiento complejo. Dicho autor ha propuesto superar las dicotomías y divisiones preexistentes en la ciencia tradicional, partir de la ruptura de las ideas metodológicas tradicionales planteadas por Aristóteles, Kant y Descartes.

El mundo es abatido por ideologías que avanzan en el transcurrir de los años, como las raíces que penetran en la tierra fértil, haciéndola parecer indestructible, no así perfecta; en donde el pensamiento complejo cuestiona los comportamientos sociales que acaban con el bienestar colectivo, mismos que actualmente inciden en la mentalidad de la economía, donde la prioridad es satisfacer el consumismo. Y como humanos no nos hemos dado cuenta y nos falta sensibilidad para percatarnos que la necesidad del consumo no nace sino se crea por medio de la manipulación en sus diversas formas.

No obstante, la realidad es completamente diferente, nada es equilibrado, nada es constante, todo permanece en un cambio y en movimiento, desde el mundo cuántico en el que se expresan los fotones, neutrones, electrones y protones; hasta el mundo macroscópico, en el que se observa lo físico a simple vista, incluyendo el movimiento de los astros. De tal suerte, debemos comprender este universo micro y macro lleno de incertidumbre, todo está conectado, que cualquier cambio que generemos en un sistema, afectará el ambiente de otro sistema, tanto aquellos sistemas que son macroscópicos, como aquellos que son microscópicos donde habitan incluso los virus. ${ }^{15}$

En relación al crecimiento exponencial del conocimiento que puede ser alcanzado y la posibilidad para todos de saber y explicar las leyes del universo, han surgido diversas teorías con la esperanza de comprender el mundo en el que vivimos, sin embargo, ninguna se ha aproximado a esta comprensión de la realidad, como lo ha hecho la teoría del caos desarrollada en el enfoque de la complejidad. ${ }^{16}$

13 Tzoc, Juan, "Multidimensionalidad en el pensamiento de los pueblos en torno al Coronavirus", Universidad Rafael Landívar de Guatemala 3(1) 10-17. DOl: https://doi.org/10.46734/revcientifica.v2i1.7 Guatemala, 2020. Pág. 14. 14 Morin, Edgar, "Introducción al pensamiento complejo", Madrid: Paidós, 2011. Pág. 16

15 Gatica, Sergio, "La urgencia de un nuevo orden económico y político", Revista Científica Internacional, 3(1), 2020. $27-29$.

16 Orellana, Oscar, "Pandemia Económica por Covid-19", Revista Científica Internacional, 3(1), 1-9. hłtps://doi. org/10.46734/revcientifica.v2i1 .6 Guatemala, 2020. Págs. 5 y 6. 
La complejidad como ciencia revolucionaria proporciona los grados de libertad para entender por qué los pueblos, las culturas y las sociedades, siempre tendrán sus propias formas de ver la realidad, que corresponden a modos actuales de vida, formas de interpretar las causas de los acontecimientos y de vivenciar la realidad. ${ }^{17}$

La complejidad a diferencia de la construcción del conocimiento en el método de la ciencia clásica está plagada de verdades absolutas, en ese sentido, desde la complejidad podemos comprender la diversidad y riqueza de las múltiples verdades. $^{18}$

Briggs y Peat añaden el aporte del novelista Joseph Conrad con respecto al significado de la verdad, proponiendo definirla como un sentimiento vivo de complicidad con la creación, con la frágil persuasiva de solidaridad que entreteje y relaciona los vínculos de las soledades en los corazones. ${ }^{19}$ Es una especie de sentimiento compartido y de múltiples perspectivas interpretativas de las diversas y complejas interpretaciones de los corazones humanos. En conclusión, no hay una sola verdad, las verdades son múltiples y diversas, tanto como lo son las variadas y bellas formas de expresión de vida creativa en el universo y en la naturaleza.

La ciencia clásica ni reconoce la diversidad de verdades, ni el sentimiento latente, ni la convicción de solidaridad que subyacen en las múltiples verdades, puesto que además el ideal reduccionista conjuga la convicción del conocimiento absoluto que ha sido garantizado por la ciencia y sus métodos cerrados, con la coexistencia en la intención política del dominio de la naturaleza para ponerla a su servicio o para explotarla en detrimento de las múltiples formas de vida universal. ${ }^{20}$

Dichos autores citan al filósofo indio Krishnamurti, quien define la verdad como un punto sin que este tenga un lugar fijo, porque en si misma la verdad es dinámica y cambiante, no se mide con palabras y no puede ser un concepto o una idea para adquirir, y porque, además no existe un camino para llegar a la verdad. ${ }^{21}$ De igual forma, no se llega a la verdad por medio de disciplinas, lógicas o técnicas. La verdad la encuentra cada quien, de forma individual a partir de las experiencias propias de la vida, sin embargo, a pesar de ser tan diversa y compleja, la verdad es lo que nos mantiene unidos. ${ }^{22}$

Conrad afirmaba que la verdad se encuentra en cualquier lugar y cualquier momento, en las pequeñas y en las grandes cosas. Sin embargo, estamos limitados

17 Briggs, John, y David Peat, "Las Siete Leyes el Caos. Las ventajas de una vida caótica", Barcelona: Grijalbo, 1999. Pág.8

18 Delgado, Op. cit., 2008. Pág. 7.

19 Briggs, y Peat, Op. cit., 1999. Pág. 28

20 Delgado, Op. cit., 2008. Pág. 35

21 Briggs, y Peat, Op. cit., 1999. Pág., 28

22 Idem. 
en nuestra forma de ver el mundo por la concepción de ideas, opiniones y emociones sobre lo que es el significado de verdad en nuestras vidas. ${ }^{23}$

En las dimensiones de: el conocimiento humano, la vida cotidiana como proceso espiritual y como proceso material de la vida; la construcción del saber científico y tecnológico, así como las formas de ser, conocer y actuar han alterado el mundo en las dimensiones antes citadas. Manifestándose en la interconexión que existe entre la revolución en epistemología, la renovación del reduccionismo a la complejidad, el holismo ambiental y la bioética. ${ }^{24}$

El conocimiento generado por accionar de las ciencias revolucionarias permite entender que somos el resultado de ese caos que dio origen a las partículas subatómicas que, al ser destruidas, dieron origen a nuestro cuerpo, nuestro contexto y nuestro mundo. Lo anterior, explica la interconexión y relación de cada persona con los sistemas naturales, los sistemas sociales y del pensamiento que fluye y envuelve nuestras vidas. ${ }^{25}$ Asimismo, esta comprensión se manifiesta en el pensamiento de los pueblos indígenas al postular que somos parte de las estrellas, que los animales no son inferiores más bien son hermanos, que el planeta tierra es nuestra madre, y que el sol y la luna son nuestros abuelos, todos los elementos universales somos parte de un mismo sistema y de múltiples relaciones desde la autoorganización del universo hasta nuestros días.

Además, somos el resultado del caos que dio origen a las partículas subatómicas que integran nuestro cuerpo, nuestro contexto, nuestra realidad. Una prueba es saber que estamos interconectados con los sistemas de la naturaleza, de la sociedad y del pensamiento que nos rodea, y fluyen a través de nosotros, lo que hace pensar que no somos diferentes a las estrellas y los animales. ${ }^{26}$ La única diferencia es que los seres humanos nos hemos convertido en consumidores compulsivos y con tendencia a destruir nuestro propio planeta.

\section{La interpretación del caos en la Cosmovisión}

Muchos pueblos indígenas, por la incertidumbre, construyeron su mundo alrededor de los rituales y de los dioses, pero no por simple ignorancia, sino porque de esa forma interpretaron la realidad del caos en su momento.

Como dicen Briggs y Peat en sus estudios, que el caos nos invita a vivir y aceptar las múltiples incertidumbres que nos presenta la vida, y a la apertura en las dimensiones creativas que transforman nuestras vidas en la profundidad y en la armonía de la turbulencia emergente. Lo anterior nos sugiere que, en vez de resistirnos a

\footnotetext{
23 Idem.

24 Delgado, Op. cit., 2008. Pág. 7.

25 Briggs y Peat, Op. cit., 2008. Pág. 11

26 Tzoc, Op cit. Pág. 15.
} 
las incertidumbres de la vida, lo que deberíamos hacer es aceptarlas, abriendo dimensiones creativas que vuelven más profundas y armoniosas la vida.

Las comunidades, las culturas, las familias y las personas, responden a un sistema de auto organización que permite constantemente un flujo de energía y material, que da forma a lo que somos y como existimos.

En tal sentido dichos autores indican que lo que somos es la manifestación de lo que nos alimentamos, del aire se respiramos, de las experiencias de nuestra vida en nuestro contexto. ${ }^{27}$ Somos parte de esa gran explosión que sigue moviéndose en vórtices que hace surgir y germinar la semilla de la tierra que nos alimenta, el agua que bebemos, el fuego que nos calienta, la tierra donde vivimos y el aire que respiramos.

Todo esto se ha nutrido en la compleja molécula de ADN que contiene las informaciones de nuestra existencia, exactamente como los diversos espacios, formas y manifestaciones que la naturaleza ha creado en cada realidad con su propia verdad.

En esa línea de pensamiento, en la cosmovisión de los pueblos indígenas, la enfermedad es concebida como una entidad, un ser, un elemento con vida, y demás, que para poderlo interpretar y comprender, ha sido necesario que tome forma humana. Con este criterio se considera que tiene espíritu, sentimiento, emociones, necesidades y deseos. Es decir que adquiere conductas humanas que hace que el ser, en este caso la enfermedad o el elemento que contiene el cuerpo, capaz de nutrirse, caminar, escuchar y por supuesto observar y sentir. ${ }^{28}$

Lo anterior explica la manera de como los pueblos indígenas construyeron sus propios conceptos y conocimientos, basado en la realidad y el lenguaje dominante de aquel momento, y la existencia se explica más que con las palabras, con la misma vivencia de la existencia, explicada de una manera que impregna su experiencia de vida.

En ese sentido, Delgado señala que los grandes logros y avances del saber han buscado la manera de crear y disponer de innovadores instrumentos para el trabajo, de la emancipación de los seres humanos de la naturaleza, de producir tecnologías para la reproducción, protección y preservación de la vida y de otras alternativas potenciales de producción y reconstrucción social. ${ }^{29}$

En la actualidad, la taxonomía de los campos de ARN, utilizando las técnicas para la separación de los cromosomas han descubierto que anteriormente las especies se agruparon en un mismo género, conocimiento que ha validado y re-

27 Briggs, y Peat, Op. cit., 1999. Pág. 23.

28 Tzoc, Op cit. Pág. 15.

29 Delgado, Op. cit., 2008. Pág. 27. 
chazado los conceptos biológicos de generación de vida y ha aportado nuevas potencialidades reproductivas y de construcción social. ${ }^{30}$

El conocimiento se ha revolucionado, crecido y desarrollado, sin embargo, tanto la ciencia, como la tecnología muchas veces se vuelven depredadoras y vulneran a las sociedades desde el momento que estos se mueven dentro del caos en dirección a determinadas intenciones que no responden a la evolución del sentido de humanidad.

De la misma forma que los ríos crean sus propios cursos indeterminados con sus turbulencias, al igual el tiempo atmosférico produce situaciones impredecibles, el efecto mariposa no es más que la ley de la influencia sutil que tiene un sistema complejo sobre otro, donde los cambios grandes o pequeños que suceden en un punto, pueden generar efectos en otro punto diferente. ${ }^{31}$ Es así como, ha humanidad puede emplear el conocimiento para que de forma sutil la convivencia de los sistemas naturales y sociales se desarrolle sin afectar la vida y su diversidad.

Intervenir en los sistemas con intenciones de servirse de la naturaleza da lugar a situaciones de caos con respuestas divergentes no esperadas, como cuando en una comunidad, sus habitantes deciden exterminar la población de serpientes; está comprobado que posteriormente tendrán que sufrir los efectos devastadores de la sobrepoblación de ratas o de ranas en los cultivos. Es así, que afectar a los elementos de un sistema es afectarnos a nosotros mismos.

Lo anterior nos aproxima a entender que formamos parte de un todo, donde cada partícula influye en la dirección y vibración de otras partículas de nuestro sistema. ${ }^{32}$ En el pensamiento de los pueblos indígenas han resaltado que cada ser humano es solamente un parte del universo, que las estrellas y los animales son sus hermanos mayores y menores, lo que significa que existe relación de afinidad entre los seres humanos y la naturaleza, por ser sistemas con relaciones y conexiones mutuas.

Cada pueblo ha aprendido a cultivar los conocimientos y han buscado diferentes formas como la experimentación, la práctica, la contemplación y la trascendencia, esta última corresponde al dominio de la mente, por ejemplo, cuando se practican técnicas de psicoterapias tradicionales para la sanación, se trabaja con el material confuso atrapado en el inconsciente.

En ese sentido, podría decirse que el conocimiento surge de la creatividad y construcción del ser humano en relación con el resto de creaciones universales, ${ }^{33}$ por cuanto que toda actividad realizada por los seres humanos, hace posible aquello que la naturaleza no realiza por sí misma, como por ejemplo la rueda,

30 Idem.

31 Briggs, y Peat, Op. cit., 1999. Pág. 56.

32 Ibidem. Pág. 76.

33 Delgado, Op. cit., 2008. Pág. 29

https://revistavidacunori.com 
el ordenador, los muebles, etc., es decir que, esa dialéctica que se da entre los elementos de la naturaleza y las acciones de los humanos, son atribuciones de valor y poder absoluto que la ciencia clásica se han atribuido al saber científico y tecnológico, y que justifican el trabajo humano, sin embargo, la perversión en este enfoque consiste en que la naturaleza está al servicio de los seres humanos para ser explotada.

Por otra parte, las invenciones humanas en interacción con la naturaleza son producto de un estado de emergencia, en esta situación la creatividad aflora cuando estamos en un estado psicológico cambiante, donde los grados de libertad de amplifican y vivimos una experiencia de la verdad, momento en el que surge y se revela el auténtico yo, ${ }^{34}$ es así como explica la teoría del caos, la razón de como las personas y los pueblos buscan las salidas o soluciones más adecuadas a los problemas, de acuerdo a la realidad y situación que se vive.

De esa forma, se puede inferir que los procesos y sistemas caóticos pueden llevar a encontrar estructuras más claras y regulares que den inicio a otro proceso, siempre complejo, pero más entendido.

Solamente de esta manera se han podido desarrollar los diferentes conocimientos que se han construido con la práctica y el valor de la existencia de la creatividad, puesto que en una sociedad nos nutrimos de energía y de las múltiples creaciones de cada la persona, de igual forma esta interacción social compartida brinda el espacio para la construcción de valores, principios éticos, del sentido colectivo y del significado de nuestra realidad. ${ }^{35}$ Este tipo de sabiduría, que han elaborado y ha servido para conducir la vida de los diferentes pueblos, es una sabiduría tradicional, que ha surgido entonces del caos para que emerja la creatividad, adoptando estrategias diversas y atrevidas, en situaciones que ha, llegado a desbordar respuestas creativas ante la emergencia.

Brigss y Peat indican que en el proceso creativo se presenta la fluidez sin intervención de la autoconciencia, la sensación de tiempo desaparece porque el desarrollo de la actividad creativa absorbe por completo. El momento de creatividad es comprendido intensamente con certeza. La fluidez en la creatividad excita por la recompensa en el avance de la creación que se está produciendo. Y experimentar el caos es una sensación agradable de ideas y relaciones múltiples. ${ }^{36}$

La ciencia clásica define la creatividad como un constructo complejo, donde un solo hemisferio cerebral interviene, valora únicamente la cantidad de la fluidez y la calidad de la originalidad en y de las ideas, dando una explicación atribuida a la biología y explicándola como un proceso de activación en redes neuronales y regiones cerebrales. Asimismo, la reduce a recuperación de información almacenada en la memoria. Como se observa, eses una definición simple e instrumental

34 Briggs, y Peat, Op. cit., 1999. Pág. 27.

35 lbídem. Pág. 19.

36 Briggs y Peat, Op. cit., 2008. Pág. 35. 
sobre el funcionamiento orgánico, no explica las múltiples interacciones que se producen en la fluidez de la creatividad como, por ejemplo, la realidad, la experiencia de vida, la cotidianeidad, la cultural, la exposición ante la emergencia o la fascinación por la vida.

Desde la ciencia clásica, podemos observar la segmentación del conocimiento, atribuyendo la creatividad de todo el proceso creativo a un solo órgano, el cerebro. Sin embargo, se deja de lado los sentidos, las sensaciones, los sentimientos, los demás órganos y sistemas que conforman el cuerpo humano, así como la interacción del contexto, donde todos y cada una de las partículas somos parte del todo, del sistema que conformamos. El proceso creativo no se propicia ni se da en un solo órgano, es una interacción de relaciones complejas.

Los sistemas sociales: humanos, naturaleza e inteligencia artificial generan la creatividad en el caos, porque el caos es la expresión creativa de la naturaleza.

Nuestros cuerpos conforman sistemas caóticos abiertos, que permiten y generan constantemente respuestas creativas a los cambios ambientales y otros. Es así, como, por ejemplo, el sistema corporal se autoorganiza en las transformaciones frágiles y en su capacidad de relacionarse y de percibir lo material y lo energéti$\mathrm{CO}^{37}$

Para el ser humano el significado de creatividad es complejo y dista de nuestro conocer y de lo que comúnmente estamos familiarizados o de lo cotidiano, es llegar a la verdad de las cosas, porque en ese camino a la verdad es donde aparece el caos. ${ }^{38}$ Esto no es algo fácil, en una sociedad donde estamos condicionados a determinadas respuestas, inclusive a una sola verdad. La creatividad son múltiples respuestas y múltiples verdades relacionadas más allá de nuestro comprender.

La creatividad ha sido reconocida desde tiempos y culturas ancestrales, y cobra importancia el reconocimiento de otras formas del saber establecido por las culturas predecesoras que actualmente conservan y manifiestan en la cotidianeidad de los pueblos que en occidente denominan subdesarrollados por la influencia homogeneizadora del saber científico moderno que excluyen a los saberes comunitarios de vida y a la socio diversidad, porque desde su perspectiva escapan o carecen de la fundamentación científica.

En ese sentido, en la creatividad y en la vida en la naturaleza podemos encontrar manifestaciones prácticas enfocadas a la reintegración del saber y resguardo de la diversidad en la vida comunitaria, así como reconocer su sociodiversidad. ${ }^{39}$

37 Briggs, y Peat, Op. cit., 1999.Pág. 26.

38 Idem.

39 Delgado, Op. cit., 2008. Pág. 18. 
La teoría del caos nos enseña un espejo reflejado en una sociedad que ocupa espacio donde las interacciones de cada pueblo, cada persona se ve relaciona íntimamente al mundo orgánico como una sola pieza que pareciera tan solo un aleteo de mariposa sin efecto alguno, pero que está conectado a una revolución de movimientos, a cientos de millas de distancia, que enmarcan las decisiones de nuevas perspectivas sociales.

Brigss y Peat relatan que los indígenas americanos han realizado practicas para inducir a la autorganización psíquica para la creatividad, esto consiste en emplear técnicas que conducen a la alucinación, se desarrollan en ambientes enclaustrados, de oscuridad y con calidez. Similarmente en las sesiones psicoanalíticas del mundo occidental, la persona es conducida a realizar libres asociaciones para luego entrar en situaciones de caos que se encuentran atrapadas en el inconsciente, de donde siempre existe algo verdadero que emerge para autoorganizarse. ${ }^{40}$

Podemos apreciar similitud en el relato mitológico del Popol Vuh al mencionar el viaje de los gemelos Junajpu e Xbalamke a Xibalbá, donde sostienen varias luchas con los dioses del inframundo.

Este relato lo asociamos con el proceso de desarrollo de la creatividad, donde debemos descender y luchar con nuestros demonios de acomodación y cotidianeidad social, emocional, psíquica para trascender y comprender la creatividad en la naturaleza. Y luego de morir en nuestras vivencias clásicas, renacemos como la caña de maíz renace en el patio de la abuela Ixmucané, en nuevas asociaciones y desarrollo de nuestra creatividad que como seres humanos poseemos.

Brigss y Peat relatan que la tribu de los pies negros realiza un proceso de apertura creativa consistente en un círculo de conversación, que representa el centro de la organización comunitaria. Es el espacio social y físico donde se toman las decisiones colectivas, se caracteriza por dejar un lugar para que al menos una persona nueva pueda entrar a aportar. En este círculo de conversación, la apertura al flujo siempre está abierta a la autoorganización. ${ }^{41}$

En la ceremonia maya sucede de forma similar, se conversa y dialoga con los abuelos representados en la energía universal, se ofrenda materialmente al fuego lo que se posee, se observa y se lee en el fuego el dialogo con la creatividad. En la creatividad de la naturaleza se encuentra el desarrollo de las ideas y nuevas formas de ver el caos de la vida.

Las culturas indígenas agrarias, mantienen una vida normal construida sobre la fortaleza y aprendizaje metafórico relacionado a la naturaleza. Briggs y Pet indican que en estas cultural la vivencia es más cercana a las resonancias caóticas

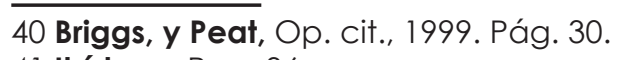

41 Ibídem., Pag. 36. 
reveladas por el espíritu de la vida de la naturaleza, ${ }^{42}$ evocando transformación de diseños que fluyen de las emociones, de los pensamientos y de la naturaleza. Este escape de energía se convierte, se refleja o se materializa en los distintos tipos de artes, de acciones y experiencias propias de vida. ${ }^{43}$

En este sentido de la vida misma se contempla el comportamiento de las conexiones culturales donde el puer y el senex luchan por permanecer siempre en la juventud, donde todos los pueblos permanecen en armonía, en la misma creatividad nace la simplificación de la vida, buscar esa verdad en lo simple, que nace en la dignificación de cada ser humano, valorando su creatividad, conectándolo con la naturaleza y recreando la satisfacción de las verdaderas necesidades que van desde lo espiritual hasta el comportamiento diario y no investido por una capa llena de posibles necesidades que debe ser satisfechas para ser feliz.

En sistema del caos las interconexiones fluyen entre elementos individuales y colectivos con una perspectiva ambivalente donde la civilización busca la verdad, la verdad de ser humano como sociedad donde cada pueblo, nación, estado entrelaza conocimientos y productos, donde se busca el bien común, en un ideal pragmático, pero donde existen de forma impuesta las ideologías de poder, jerarquías y competencia por encima de sus tendencias naturales a la actividad creativa colectiva.

Sin embargo, al valorar la creatividad como un fenómeno raro y algo anormal es importante considerar que están desapareciendo las formas ancestrales de la vida humana, derivado por la constante transformación, homogeneización y crecientes formas de dependencia. La vida cotidiana conlleva a la destrucción de las formas de vida, donde la instrumentalización está condicionada como un modo material y único de realización de la vida .

\section{La Vida y el Sentir desde la Naturaleza}

En la teoría del caos, Briggs y Peat nos indican que podemos profundizar en cuanto a las intuiciones que afectan nuestra naturaleza de seres creativos. Sin embargo, se reconoce que la sociedad está conformada de muchas personas desinformadas con respecto a la creatividad, porque la entienden como un don especial asignada a unos pocos y que su principal objetivo es crear algo nuevo, actualmente esto es uno de los grandes mitos reduccionistas. ${ }^{44}$

Asimismo, la creatividad en la naturaleza es antagónica a la homogeneización que actualmente se promueve a gran escala en los sistemas sociales, porque la homogeneización dirige el decrecimiento de la diversidad en el aspecto espiri-

42 Ibídem., Pág. 161.

43 Delgado, Op. cit., 2008. Pág. 18.

44 Briggs, y Peat, Op. cit., 1999. Pág. 158.

https://revistavidacunori.com 
tual del ser humano, para excluir y marginar a las personas o a las manifestaciones vivientes en el universo. ${ }^{45}$

Brigss y Peat indican que se cree que los dotados de creatividad son seres un poco locos, se relaciona íntimamente a la demencia y a la creatividad, como signo de anormalidad humana. ${ }^{46}$ Entonces, la creatividad que nos permite construir conocimiento complejo es considerada y definida peyorativamente por el enfoque clásico.

Comúnmente se considera que la creatividad significa un camino para ir más allá en dirección hacia lo desconocido, es la búsqueda de la verdad de las cosas y en ese camino es donde experimentamos la experiencia del caos. ${ }^{47}$ Este camino, presenta un sinfín de indeterminaciones y vicisitudes donde la comprensión de las relaciones de los sistemas es más fluida, asimismo, se establecen aquellas que a simple vista han sido obviadas, pero que han estado ahí frente a nuestras narices y que son las respuestas en los estados emergentes.

Brigss y Peat agregan que cuando somos creativos en nuestra vida o trabajo y estamos sumergidos en el caos algunas veces emerge la bifurcación, de donde se logra que aparezca la creación con fluidez y sin obstáculos. ${ }^{48}$

Las formas de creatividad que se manifiestan en la naturaleza van más allá de la música, la pintura, el teatro o las matemáticas, según Briggs y Peat, porque no se percibe la creatividad en las actividades cotidianas como la observación de la naturaleza, recordar un sueño, conversar con alguna persona o simplemente ver una obra artística. La creatividad está en toda la vida de la naturaleza y de las interacciones de los sistemas sociales humanos y naturales. ${ }^{49}$

La creatividad aparece cuando abandonamos las estructuras que conocemos y nos aventuramos en lo desconocido del caos, al sacrificar lo que nos es familiar. Briggs y Peat (1999) creen que la clave para la actividad creativa se encuentra en la autoorganización de los materiales que tenemos a nuestra disposición. Para nosotros los seres humanos esto significa que debemos crear con el material de nuestras vidas..$^{50}$

A lo anterior podemos agregar que desde la ciencia clásica la producción de conocimientos se limita a una actividad específica alejada de la vida cotidiana y muchas veces opuesta a ella.

45 Delgado, Op. cit., 2008. Pág. 18.

46 Briggs, y Peat, Op. cit., 1999. Págs. 16-18.

47 Ibídem, Pág. 16.

48 Briggs, y Peat, Op. cit., 1999. Pág. 35.

49 Briggs, y Peat, Op. cit., 1999. Pág. 17.

50 Espinoza, Linda, "Un nuevo aprendizaje frente a la complejidad de la vida", Revista Académica CUNZAC, (2020). 3(1), 45-51. https://doi.org/10.46780/cunzac.v3i1.18. Guatemala, 2020.Pág. 47. 
La vida cotidiana es tomada como ente de la pasividad e ineptitud para desarrollar conocimiento que compita con el conocimiento científico. El conocimiento de la vida cotidiana se considera inferior en aspectos cognoscitivos y se desvaloriza cualquier conocimiento que se derive de esta. ${ }^{51}$

Para Briggss y Peat, la teoría del caos tiene que ver con el comportamiento creativo de la naturaleza, donde constantemente se crean nuevas formas y estructuras con su particular imprevisibilidad y confusión, como el fluir mismo de la creatividad. ${ }^{52}$

Sin embargo, la aceptación de la creatividad en la naturaleza se ve afectada por la influencia de la ciencia clásica que en la modernidad impuso la creencia absoluta en el conocimiento, la predicción y la manipulación con el ingrediente de la exactitud y para el exclusivo beneficio humano.

Lo anterior, enfrenta varios problemas entre los que destaca el ambiental, donde el conocimiento exacto, la predicción y la manipulación se hacen improbables. ${ }^{53}$ Porque la creatividad en la naturaleza emerge desde el caos y en el caos no existe ni el control, ni la manipulación, ni la predictibilidad.

La teoría del caos manifiesta la creatividad en la naturaleza y su diversidad de comportamientos plasmados en las expresiones meteorológicas atmosféricas del tiempo, en el movimiento del agua, en las interacciones neuronales y hasta en la impredecibilidad del comportamiento económico de la bolsa de valores. Asimismo, es la creación del modo de la naturaleza construyendo nuevas formas y estructuras en su imprevisibilidad y confusión. ${ }^{54}$

Actualmente los sistemas sociales: humanos y naturales se ven afectados por la constante homogeneización en de la vida de los seres humanos y en la pérdida de la diversidad social, que son para la creatividad y la naturaleza destructivas e indeseables. La perturbación en la cotidianeidad de elevados consumos en la naturaleza incrementa su fragilidad y sumado a esto la perdida de la sociodiversidad en la búsqueda de estados sociales homogéneos y de equilibrio, equivalen al camino hacia la eliminación de la vida y de la naturaleza. ${ }^{55}$

El sistema del dominio de la naturaleza compaginada con el capitalismo originario de la economía política ecológica propone mantener un equilibrio de riqueza para el que la desee, sin dañaos a los más pobres y necesitados, cosa que en la realidad no se ha dado. El nuevo orden económico solidifica el valor como un agregado a la política económica pero no es más que un ladrón de bancos donde saquean las bóvedas repletas de tesoros y riqueza de la naturaleza.

51 Delgado, Op. cit., 2008. Pág. 41.

52 Briggs, y Peat, Op. cit., 1999. Pág. 18.

53 Delgado, Op. cit., 2008. Pág. 7.

54 Briggs, y Peat, Op. cit., 1999. Pág. 18.

55 Delgado, Op. cit., 2008. Pág. 18. 
Hoy por hoy, la conservación es un principio muy eficiente, donde la eficiencia juega un papel primordial, se confunde el tiempo con la eficiencia, sin contar la participación humana; atribuyendo a la eficiencia el valor del tiempo mismo. ${ }^{56}$

Sin embargo, la situación provocada por el Coronavirus, también ha incidido significativamente en la vida en la naturaleza, y en el ser humano ha provocado la reflexión sobre las posibilidades que tenemos en el universo para la regeneración de las múltiples manifestaciones de vida, donde la naturaleza nos ha brindado nuevamente una obra creativa. Se reconoce este caos que vive el sistema, las múltiples renovaciones en el aire, agua, vida animal, vidas humanas, entre otros. ${ }^{57}$

La representación del caos en la naturaleza, es la creatividad manifiesta en el sistema natural. Los seres humanos somos naturaleza y nuestros cuerpos están conformados de sistemas caóticos abiertos, que constantemente responden creativamente a los cambios ambientales.

Un ejemplo de esto es la capacidad de conexión del cerebro, de auto organizarse en cada uno de los actos de percepción. Además, podemos observar, que la naturaleza dispone de diversos y diferentes modos en cuanto al principio del caos auto organizado para ser usado y para responder a los mecanismos de nuestra subsistencia..$^{58}$

Las personas dedicadas a actividades relacionadas con la creatividad son bastante receptivas para comprender cómo el caos emerge, se reconocen en sí mismos como colaboradores del caos. Al observar la forma en que los creadores profesionales trabajan con el caos, nos permite apreciar un proceso dispuesto al alcance de cualquiera de nosotros, porque todos somos creativos, según lo refiere Briggs y Peat. ${ }^{59}$

También, añaden que la creatividad es un proceso lento, se da al explorar las incertidumbres, al atrevernos a sacrificar lo que vemos como familiar. La creatividad es tan impredecible de aparecer, de pronto en un diálogo, en una acalorada discusión o en la interacción del canje de ideas que permite iluminar una conversación innovadora o una autentica forma siempre de expresión.

La creatividad puede aparecer y fluir en la contemplación de un árbol, al apreciar nuestro conocimiento sobre este o al observarlo con sus múltiples bifurcaciones formadas por ramos, nudos y laberintos, cuando en su interacción con el aire y la luz se aprecia su única y sutil forma de vida. ${ }^{60}$

\footnotetext{
56 Ibidem. Pág. 144.

57 Castillo, Nancy, "El Fenómeno del Coronavirus y el Biodesarrollo: sus consecuencias y oportunidades", Revista Ciencia Multidisciplinaria CUNORI. Guatemala, 2020. Pág. 129.

58 Briggs, y Peat, Op. cit., 1999. Pág., 26.

59 Idem

60 Ibidem, Pág. 30.
}

https://revistavidacunori.com 
Cuando todo eso sucede en nuestro sentir y cambia nuestra forma de ver, la intuición creativa se autoorganiza y nos agarra desprevenidos ante el gozo de la verdad o la esencia inesperada, que para nosotros estuvo oculta en la intimidad de los objetos de nuestro mundo familiar ordinario que nos ha rodeado hasta ese momento. 61

\section{Reflexiones}

Se llega a comprender que para ser creativos es necesario sentir e intuir el saber y el no saber, transitando en lo no adecuado, en la incertidumbre, en la incomodidad, en la alegría, aceptando las diversas caras de las múltiples formas de la no linealidad; en otras palabras, experimentar la multiplicidad de fases en la creatividad del caos. ${ }^{62}$

La actitud común ante los errores es una respuesta de desprecio al error, siempre intentamos prever de forma determinista que nuestras acciones no sufran contratiempos y las fallas son consideradas motivo de vergüenza. Sin embargo, desde la perspectiva de caos, las personas creativas reconocen en el error la planificación de un experimento para transformarse en una oportunidad de conocer algún ámbito de una verdad que amplifica y apertura el espacio para la fluidez del instante creativo. ${ }^{63}$

En conclusión, los sistemas sociales: humanos, naturaleza e inteligencia artificial vivimos y experimentados constantemente en interacción, generando vida y creatividad diversa. Los condicionamientos sociales, las verdades únicas, los métodos científicos clásicos, la segmentación y otros elementos pensados con límites y fronteras, muchas veces provocan restrictivas perceptivas al maravilloso, armonioso y bello mundo creativo donde el caos que se nos presenta día a día y en el que vivimos constantemente es la vida misma.

\section{Referencias Bibliográficas}

Briggs, John y Peat, David, "Las Siete Leyes el Caos. Las ventajas de una vida caótica", Grijalbo. Barcelona, 1999.

Castillo, Nancy, "El Fenómeno del Coronavirus y el Biodesarrollo: sus consecuencias y oportunidades", Revista Ciencia Multidisciplinaria CUNORI. Vol. 4 No. 2. Págs. 125-132 Guatemala, 2020. Recuperado de: https://revistacunori.com/index.php/ cunori/article/view/136/192

61 Briggs y Peat, Op. cit., 1999. Pág. 31.

62 Idem.

63 Idem.

https://revistavidacunori.com 
Delgado, Carlos, "Hacia un nuevo saber, La bioética en la revolución contemporánea del saber", Universidad el Bosque. Colombia, 2008.

Espinoza, Linda, "Un nuevo aprendizaje frente a la complejidad de la vida", Revista Académica CUNZAC, 3(1), 45-51. Guatemala, 2020. https://doi.org/10.46780/ cunzac.v3il.18

Gatica, Sergio, "La urgencia de un nuevo orden económico y político", Revista Científica Internacional, 3(1), 2020. p. 27-29. https://doi.org/10.46734/revcientifica.v2il.9

Morin, Edgar, "La Vía, para el futuro de la humanidad", Editorial Paidós. Madrid, 2011.

Morin, Edgar, "Introducción al pensamiento complejo", Editorial Paidós. Madrid, 2011.

Orellana, Oscar, "Pandemia Económica por Covid-19", Revista Científica Internacional, 3(1), 1-9. https://doi.org/10.46734/revcientifica.v2i1.6 Guatemala, 2020.

Tzoc, Juan, "Multidimensionalidad en el pensamiento de los pueblos en torno al Coronavirus", Universidad Rafael Landívar de Guatemala 3(1) 10-17. DOl: https:// doi.org/10.46734/revcientifica.v2i1.7 Guatemala, 2020.

\section{Biografía de los autores}

Son estudiantes del Doctorado en Investigación en Educación en el Centro Universitario de Oriente CUNORI de la Universidad de San Carlos de Guatemala.

\section{Los textos publicados son responsabilidad de los autores.}

Copyright $\odot$ 2020. Los derechos son de los autores. Oscar Armando González Díaz, Juan Tzoc, Julieta Maribel López y Servio Tulio Argueta Ramos.

$$
\text { (c) } \underset{\mathrm{BY}}{\mathrm{BY}}
$$

Los textos están protegidos por una licencia Creative Commons 4.0 Internacional Usted es libre de compartir, copiar y redistribuir el material en cualquier medio o formato y adaptar el documento, remezclar, transformar y crear a partir del material, siempre que cumpla con la condición de atribución, debe reconocer el crédito de la obra de manera adecuada.

El manuscrito es de acceso abierto 